\title{
A case study of large-amplitude ULF waves in the Martian foreshock
}

\author{
LiCan Shan 1,2*, YaSong Ge1,2,3, and AiMin Du1,2,3 \\ ${ }^{1}$ Key Laboratory of Earth and Planetary Physics, Institute of Geology and Geophysics, Chinese Academy of Sciences, Beijing 100029, China; \\ Innovation Academy for Earth Science, Chinese Academy of Sciences, Beijing 100029, China; \\ ${ }^{3}$ College of Earth and Planetary Sciences, University of Chinese Academy of Sciences, Beijing 100049, China
}

Key Points:

- Foreshock ULF waves are identified upstream of the Martian bow shock

- The property of ion density varying in phase with perturbations of wave field indicates that it is a fast mode wave

- The foreshock waves are excited by interactions between solar wind and backstreaming ions through right-hand beam instability

Citation: Shan, L. C., Ge, Y. S., and Du, A. M. (2020). A case study of large-amplitude ULF waves in the Martian foreshock. Earth Planet. Phys., 4(1), 45-50. http://doi.org/10.26464/epp2020004

\begin{abstract}
Foreshock ultralow frequency (ULF) waves constitute a significant physical phenomenon in the plasma environment of terrestrial planets. The occurrence of these waves, associated with backstreaming particles reflected and accelerated at the bow shock, implies specific conditions and properties of the shock and its foreshock. Using magnetic field and ion measurements from MAVEN, we report a clear event of ULF waves in the Martian foreshock. The interplanetary magnetic field connected to the Martian bow shock, forming a shock angle of $\sim 51^{\circ}$. Indicating that this was a fast mode wave is the fact that ion density varied in phase with perturbations of the wave field. The peak frequency of the waves was about $0.040 \mathrm{~Hz}$ in the spacecraft frame, much lower than the local proton gyrofrequency $(\sim 0.088 \mathrm{~Hz})$. The ULF waves had a propagation angle approximately $34^{\circ}$ from ambient magnetic field and were accompanied by the whistler mode. The ULF waves displayed left-hand elliptical polarization with respect to the interplanetary magnetic field in the spacecraft frame. All these properties fit very well with foreshock waves excited by interactions between solar wind and backstreaming ions through right-hand beam instability.
\end{abstract}

Keywords: foreshock ULF waves; Martian bow shock; backstreaming ions

\section{Introduction}

Planetary foreshock regions, characterized by interactions between backstreaming particles and ultralow frequency (ULF) waves, are different from interplanetary solar wind. There are several different types of backstreaming ion distributions in the foreshock region, i.e., field-aligned beam (FAB), and gyrating and diffuse ion distributions, which have been identified by previous studies (e.g., Gosling et al., 1978; Bonifazi and Moreno, 1981; Fuselier, 1995). An FAB generally originates from a quasi-perpendicular shock, existing near the ion foreshock upper boundary. The FAB propagates upstream along the interplanetary magnetic field (IMF) at a bulk speed of a few keV with a very limited spread in pitch angle (Meziane et al., 2005). In the FAB region, no ULF waves have been observed by spacecraft (Paschmann et al., 1979). Gyrating ions appear field-aligned, but have a phase space density peak with a large spread in pitch angle (Fuselier et al., 1986). These ions have frequently been observed downstream of the FAB distributions, and they extend approximately from 9 to $83 R_{\mathrm{E}}$ at the Earth (Meziane et al., 2001). Sometimes gyrating ions and the FAB are

Correspondence to: L. C. Shan, Icshan@mail.iggcas.ac.cn

Received 09 NOV 2019; Accepted 19 JAN 2020.

Accepted article online 21 JAN 2020.

C 2020 by Earth and Planetary Physics. observed simultaneously due to the large gyration orbit of the ions close to the FAB-gyrating ion boundary (Meziane et al., 2004). Investigations in detail show that gyrating ions can be produced from an $F A B$ through nonlinear wave-particle interactions (Mazelle et al., 2000) at the edge of the gyrating ion region and the boundary of the foreshock ULF waves (Mazelle et al., 2007). These ions are bunched in gyrophase angle when the associated ULF waves are quasi-monochromatic and have large amplitudes $(\delta B / B \sim 1)$ (Meziane et al., 2001; Mazelle et al., 2003). Diffuse ions are frequently observed upstream of a quasi-parallel bow shock. They are usually accompanied by non-monochromatic ULF waves (Hoppe et al., 1981).

Linear theory and simulation results reveal that FABs are very effective in driving ion/ion right-hand resonant instability, which is responsible for foreshock wave generation (Gary et al., 1981; Hoshino and Terasawa, 1985; Winske and Quest, 1986; Gary, 1991). In the terrestrial foreshock several distinct waves are identified (Fairfield, 1969; Le and Russell, 1994; Greenstadt et al., 1995; Burgess, 1997; Wilson III, 2016). The most significant wave activity is the ULF wave, with a typical period of $\sim 30 \mathrm{~s}$, which has been observed extensively in the Earth's foreshock (Hoppe and Russell, 1983; Thomsen et al., 1985; Le and Russell, 1992; Meziane et al., 2001; Mazelle et al., 2003; Eastwood et al., 2005a, b) and other ter- 
restrial bodies, such as Saturn (e.g., Bertucci et al., 2007; Andrés et al., 2013), Mercury (e.g., Le et al., 2013) and Venus (e.g., Shan et al., 2014). The foreshock is dependent on the shock structures and the IMF. Foreshock wave generation and evolution/propagation depend importantly on the size of the foreshock space compared to the ion gyroradius. At Mars, few foreshock ions have been identified because, compared to the ion gyroradius, the Martian shock and its foreshock are small. As a result of the planet's nonmagnetic body, some Martian parameters are very different from those at the Earth (Yamauchi et al., 2011), resulting in some interesting physical phenomena in the Martian foreshock (Mazelle et al., 2004). Using magnetic field and ion data measured by MAVEN instruments, we have identified a clear event of foreshock ULF waves at Mars. This implies that the foreshock space at Mars is large enough for an ion-ion right-hand beam instability to generate foreshock ULF waves by backstreaming ions. More detailed properties of foreshock ULF waves should be investigated in the future by statistical research.

\section{Observations}

The MAVEN spacecraft was launched on 18 November 2013 and arrived in Mars orbit on 21 September 2014 (Jakosky et al., 2015). The spacecraft has an elliptical orbit (period $\sim 4.5 \mathrm{~h}$ ) with a periapsis of $\sim 150 \mathrm{~km}$ and an apoapsis of $\sim 1.8 R_{\mathrm{M}}$ (Mars radius $\sim 3397 \mathrm{~km}$ ). Figure 1 shows 10 min interval observations of the foreshock ULF waves measured by MAVEN on 14 August 2015. The spacecraft passed through the outbound bow shock, crossing at $~ 22: 04: 20$ UT (not shown here). During this interval of interest, the space- craft traveled from the position $(1.23,0.63,2.53) R_{\mathrm{M}}$ to $(1.38,0.78$, $2.35) R_{\mathrm{M}}$ in the Mars-centered Solar Orbital (MSO) frame, where the $x$ direction is along the Mars-Sun line; $y$ is aligned with the dusk direction, and $z$ completes the right-handed coordinate system. Magnetic field data, measured by a MAG instrument (Connerney et al., 2015), show large-amplitude waves upstream of the Martian bow shock. The maximum peak-to-peak amplitude in the wave front is as large as $\sim 8 \mathrm{nT}$. The average magnetic field is about $(5.2,-1.7,0.8) \mathrm{nT}$, and the IMF cone angle (the angle between the IMF line and the $x$-axis of MSO) is $\sim 20^{\circ}$. During this interval, the IMF was connected to the Martian bow shock, and the shock angle $\theta_{\mathrm{Bn}}$ (between the IMF line and the shock normal) was about $51^{\circ}$. Here we calculated the shock normal with a modeled bow shock for Mars (Vignes et al., 2000). The solar wind flow velocity $\left(v_{\mathrm{sw}}\right)$ was $\sim 447 \mathrm{~km} / \mathrm{s}$, and the ion density $\left(n_{\mathrm{i}}\right)$ was $\sim 1.44 \mathrm{~cm}^{-3}$ observed by an SWIA instrument (Halekas et al., 2015). The magnetic magnitude varied in phase with the solar wind ion density and out of phase with the solar wind velocity. This property indicates that it was a fast magnetosonic wave in the Martian foreshock region.

Figure 2 shows the power spectrum and hodogram anslysis of the foreshock ULF waves shown in Figure 1. We separate the spectrum into transverse (red) and compressional components (black). The peak frequency, marked by an arrow, is about $0.040 \mathrm{~Hz}$. The local proton gyrofrequency represented by a dashed line is $\sim 0.088$ $\pm 0.023 \mathrm{~Hz}$ in the spacecraft frame. The frequency of the foreshock waves is much lower than the gyrofrequency. Using the Minimum Variance Analysis (MVA) method (Sonnerup and

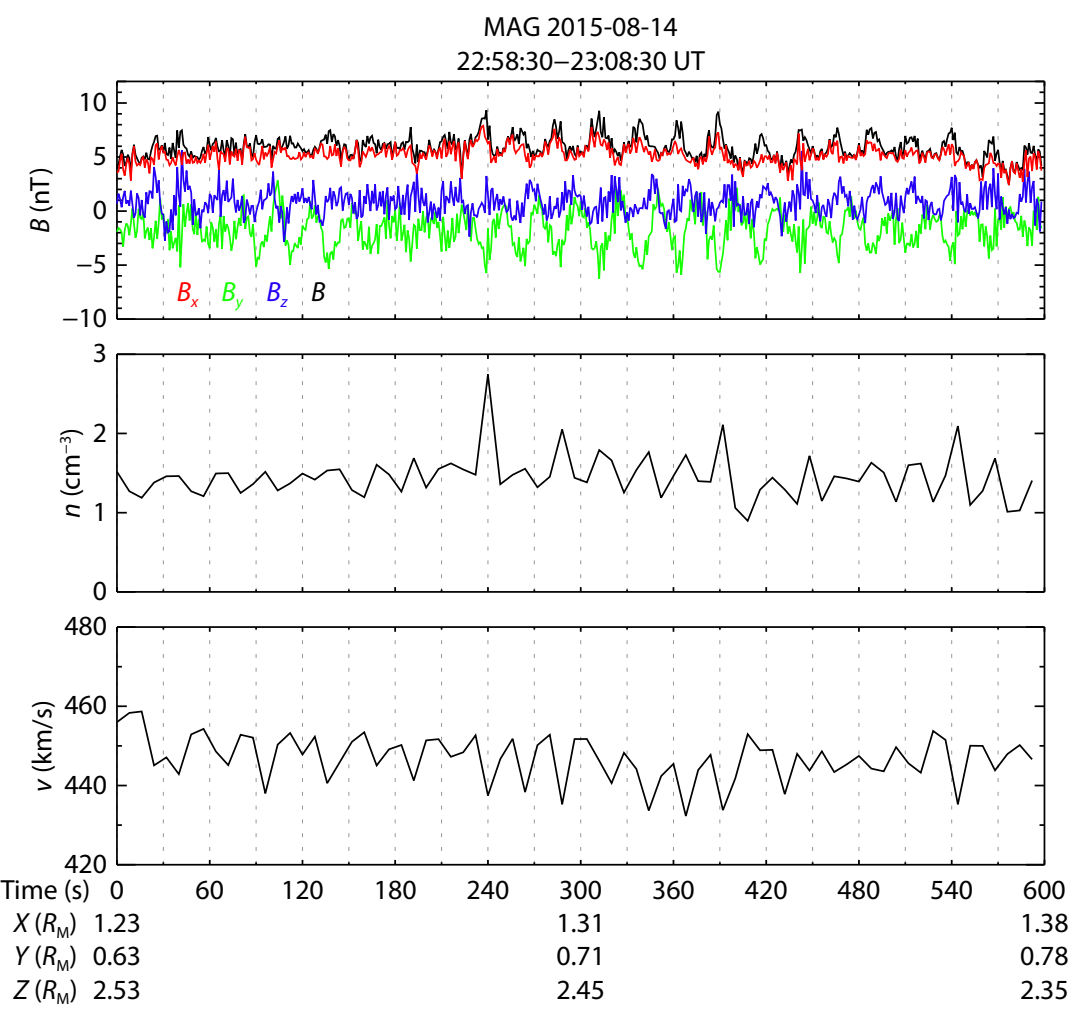

Figure 1. A ULF foreshock wave occurrence in the Martian foreshock observed by MAVEN on 14 August 2015. Magnetic field components are shown in different colors. Solar wind ion density and bulk velocity measured by the SWIA instrument. Magnetic field data sampled at $1 \mathrm{~s}$ and ion flow data sampled at $8 \mathrm{~s}$. 

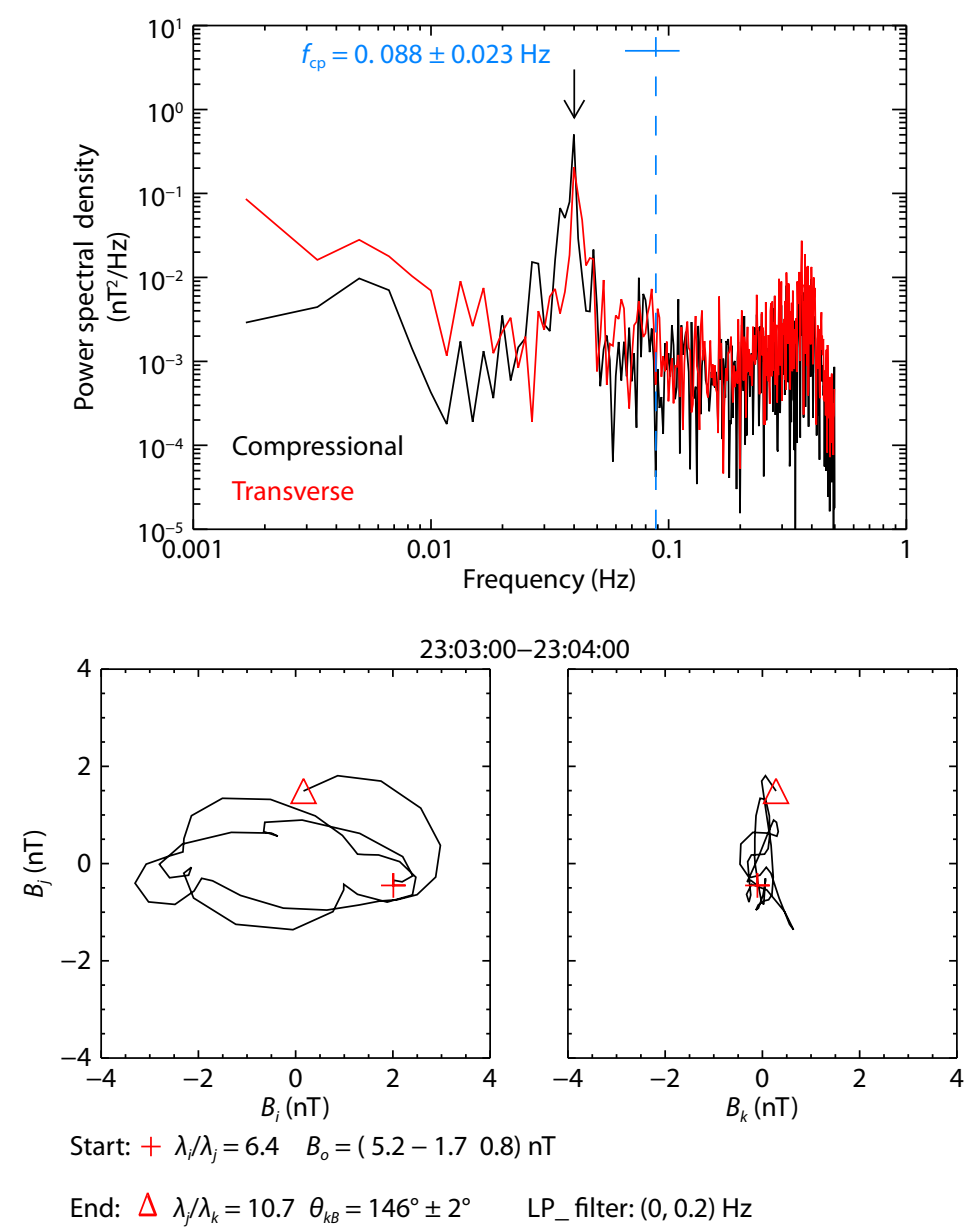

Figure 2. Wave power spectrum and hodograms for the ULF waves shown in Figure 1. The arrow and the blue dashed line mark the peak frequency of the ULF waves and the local proton gyrofrequency, respectively. In the bottom panels, plus sign and triangle show the beginning and end of the interval, respectively.

Scherble, 1998), we conducted a hodogram analysis on 1-min subintervals of the waves. It should be noted that a lowpass filter from 0 to $0.2 \mathrm{~Hz}$ was used as we performed the hodogram. $B_{i}$ and $B_{j}$ are the wave field components, and $B_{k}$ is parallel or antiparallel to the wave vector direction. The ratios of maximum to intermediate $\left(\lambda_{i} / \lambda_{j}\right)$ and the intermediate to minimum $\left(\lambda_{j} / \lambda_{k}\right)$ are 6.4 and 10.7, respectively. The latter ratio indicates the MVA is good for this case. The propagation angle $\theta_{k B}$ (angle between the wave propagation direction and the ambient magnetic field) is about $146^{\circ}$. The propagation direction has an angle of $\sim 28^{\circ}$ from the $x$ axis of MSO system. We find that the wave exhibits a left-hand elliptical polarization with respect to the background field in the spacecraft frame (bottom-left panel in Figure 2). Assuming electrons have the same temperature as ions (average value $\sim 32 \mathrm{eV}$ measured by SWIA), we estimate the phase speed $\left(v_{\mathrm{ph}}\right)$ of the fast magnetosonic wave as $\sim 126 \mathrm{~km} / \mathrm{s}$, which is much smaller than the solar wind velocity. Because of the Doppler shift by solar wind convection, the wave frequency in the solar wind frame is $f=f_{\mathrm{ob}} /\left(1+v_{\mathrm{sw}} / v_{\mathrm{ph}} \cdot \cos \theta_{\mathrm{kv}}\right)$. We then estimate the wavelength in the $x$-axis, finding it to be $\sim 3.8 R_{\mathrm{M}}$. Here $f$ and $f_{\mathrm{ob}}$ are the wave frequency in the solar wind and spacecraft frames, respectively. $\theta_{k v}$ is the angle between the wave propagation direction and the solar wind velocity. We found that the wave is right-hand polarized with respect to the average magnetic field in the solar wind plasma frame. High-frequency waves are also identified by the wave spectrum analysis. Using high-frequency $(\sim 32 \mathrm{~Hz})$ magnetic field data, we analyze the high-frequency waves shown in Figure 3. This type of wave, termed 'whistler', has also been observed in the Earth foreshock and the interplanetary medium (e.g., Russell et al., 1971; Fairfield, 1974; Tsurutani et al., 2001); whistler waves are associated with electron foreshocks (Tsurutani et al., 2001). The average wave period of the whistler wave, obtained from the power spectrum analysis in Figure 2, is about $2.7 \mathrm{~s}$. The wave propagation direction has an angle of $\sim 11^{\circ}$ from the ambient field. The whistler wave has a large amplitude. It is left-hand circularly polarized with respect to the ambient magnetic field in the spacecraft frame.

Large-amplitude foreshock ULF waves are excited by interactions between solar wind and backstreaming ion beams through an ion/ion beam instability (Gary et al., 1981). Figure 4 shows the associated ion energy fluxes in different azimuth directions in the STATIC frame (McFadden et al., 2015). During the interval from $\sim 22: 52$ to 22:53 UT, the instruments also detected a hot flow anomaly, which is one important foreshock transient (Schwartz et al., 2000). The azimuth of the solar wind beam is about $110^{\circ}$ in the STATIC instrument frame. From 22:54 UT, an abundance of back- 

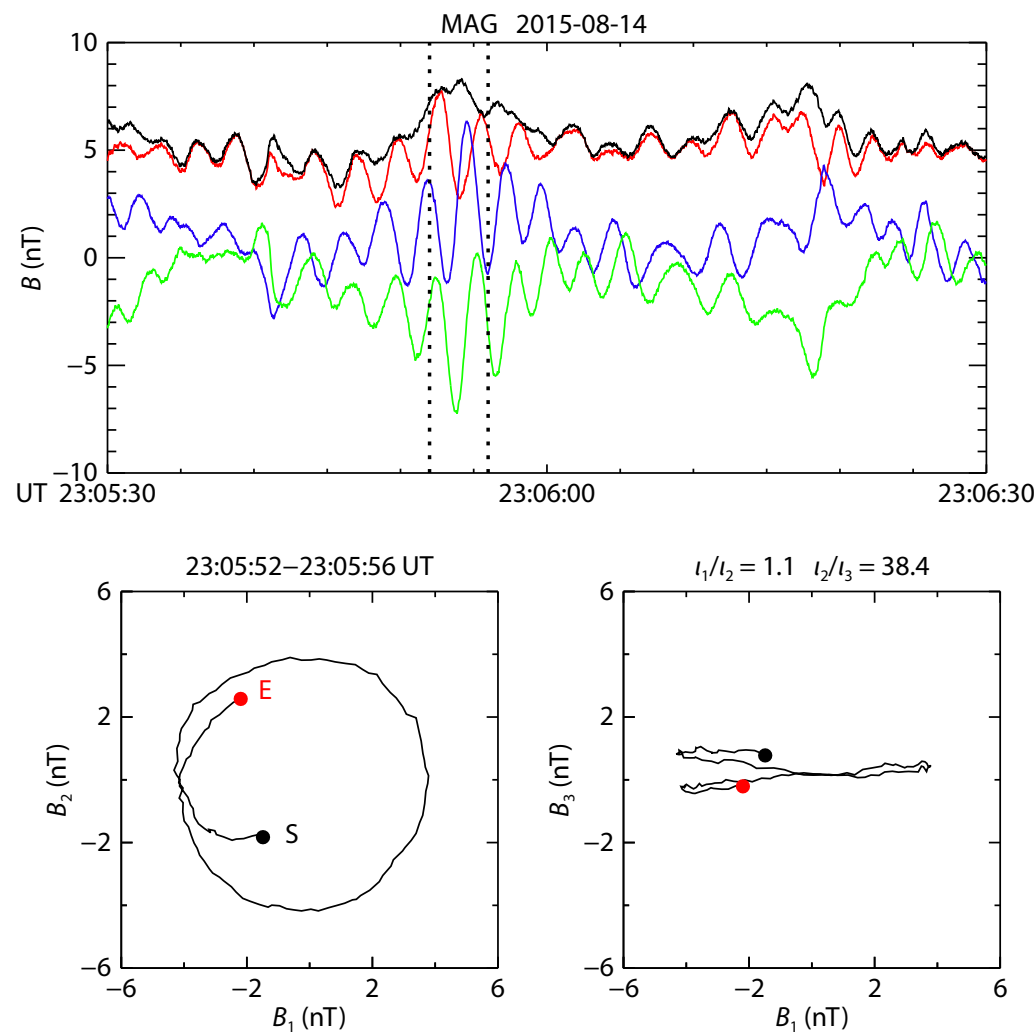

Figure 3. High-frequency waves accompanying the fast mode waves. In the top panel, high-frequency whistler waves accompanied the low frequency waves. The dotted lines mark the subinterval for hodogram analysis. In the bottom panels, ' $\mathrm{S}$ ' and ' $\mathrm{E}$ ' show the start and end of the subinterval.
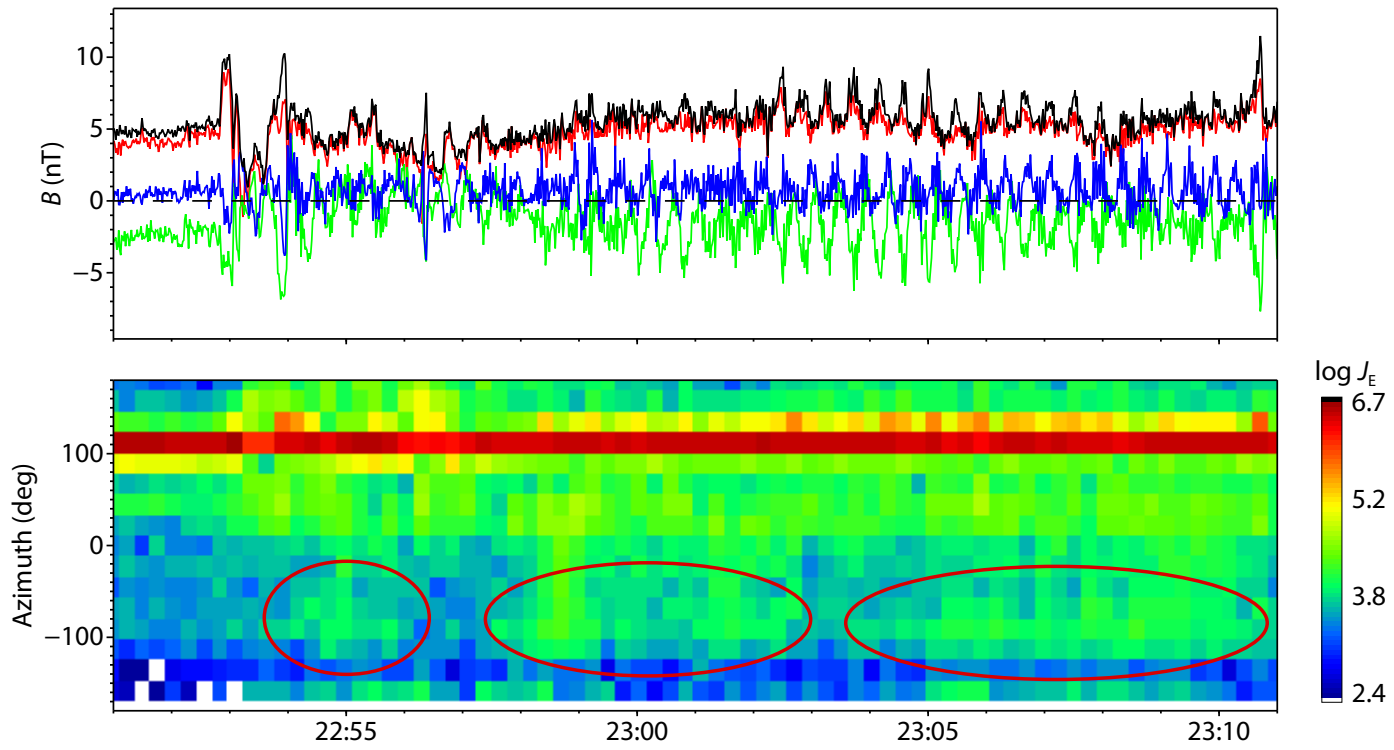

Figure 4. Foreshock ULF waves, and ion energy fluxes for different azimuth values in the STATIC frame. Ion data have a time resolution of $16 \mathrm{~s}$.

streaming ion fluxes (marked by the red ellipses) were detected. Then the ULF wavewas excited and its amplitude increased.

\section{Conclusions and Discussion}

The occurrence of foreshock ULF waves is a significant physical phenomenon at a terrestrial planet. Using magnetic field and ion data from MAVEN, we identify an event of foreshock ULF waves at
Mars. The magnetic magnitude of the waves varies in phase with the solar wind ion density and out of phase with the solar wind velocity, which is a significant signature of a fast magnetosonic wave. Based on the wave spectrum and hodogram analysis, we find that the wave period is $\sim 25 \mathrm{~s}$, which is about twice the local proton cyclotron period. The wave has a large amplitude and a propagation angle of $\sim 34^{\circ}$ from the background field. It exhibits a 
left-handed elliptical polarization with respect to the ambient magnetic field in the spacecraft frame. The phase speed of the wave $(\sim 126 \mathrm{~km} / \mathrm{s})$ is much smaller than the solar wind velocity, which means that the solar wind Doppler shift induces a reversal in the observed polarization. The estimated wavelength in the $x$ axis is $\sim 3.8 R_{\mathrm{M}}$, which is slightly larger than the foreshock waves observed at the Earth $\left(\sim 1.5 R_{\mathrm{E}}\right)$ and Venus $\left(\sim 1.35 R_{\mathrm{V}}\right)$ (Eastwood et al., 2005a; Shan et al., 2016). We suggest that wave we have studied was excited by interactions between the solar wind and backstreaming ions, through an ion/ion right-hand instability. Fast mode waves are very different from proton cyclotron waves, and the interactions with solar wind are also different (Romanelli et al., 2018). This fast mode wave is accompanied by whistler waves that have been identified previously in the Earth's foreshock (e.g., Fairfield, 1974). Different explanations have been proposed for whistler wave generation; it is believed that the whistler is associated with electron foreshock (Tsurutani et al., 2001; and references therein). Results of Meziane et al. show two populations of foreshock electrons upstream of the Martian bow shock (Meziane et al., 2017). The detailed characteristics of the foreshock ULF waves and whistler waves at Mars should be obtained from a statistical investigation.

\section{Acknowledgments}

The MAVEN data are publicly available through the Planetary Data System. L. C. Shan thanks C. Mazelle for helpful discussions and E. Penou for his assistance in data processing. This work was supported by the National Key Research and Development Program of China (2016YFB0501300, 2016YFB0501304), the National Natural Science Foundation of China (Grants No. 41774187, 41674168, 41774176), Beijing Municipal Science and Technology Commission (Grant No. Z191100004319001), the Strategic Priority Research Program of Chinese Academy of Sciences (Grant No. XDA14040404), and the pre-research Project on Civil Aerospace Technologies No. D020103 funded by CNSA.

\section{References}

Andrés, N., Gómez, D. O., Bertucci, C., Mazelle, C, and Dougherty, M. K. (2013). Saturn's ULF wave foreshock boundary: Cassini observations. Planet. Space. Sci.(79-80), 64-75. https://doi.org/10.1016/j.pss.2013.01.014

Bertucci, C., Achilleos, N., Mazelle, C., Hospodarsky, G. B., Thomsen, M., Dougherty, M. K., and Kurth, W. (2007). Low-frequency waves in the foreshock of Saturn: First results from Cassini. J. Geophys. Res. Space Phys., 112(A9), A09219. https://doi.org/10.1029/2006JA012098

Bonifazi, C., and Moreno, G. (1981). Reflected and diffuse ions backstreaming from the Earth's bow shock 2. Origin. J. Geophys. Res. Space Phys., 86(A6), 4405-4413. https://doi.org/10.1029/JA086iA06p04405

Burgess, D. (1997). What do we really know about upstream waves?. Adv. Space Res., 20(4-5), 673-682. https://doi.org/10.1016/S0273-1177(97)00455-9

Connerney, J. E. P., Espley, J., Lawton, P., Murphy, S., Odom, J., Oliversen, R., and Sheppard, D. (2015). The MAVEN magnetic field investigation. Space Sci. Rev., 195(1-4), 257-291. https://doi.org/10.1007/s11214-015-0169-4

Eastwood, J. P., Balogh, A., Lucek, E. A., Mazelle, C., and Dandouras, I. (2005a). Quasi-monochromatic ULF foreshock waves as observed by the fourspacecraft Cluster mission: 1. Statistical properties. J. Geophys. Res. Space Phys., 110(A11), A11219. https://doi.org/10.1029/2004JA010617

Eastwood, J. P., Balogh, A., Lucek, E. A., Mazelle, C., and Dandouras, I. (2005b). Quasi-monochromatic ULF foreshock waves as observed by the fourspacecraft Cluster mission: 2. Oblique propagation. J. Geophys. Res. Space
Phys., 110(A11), A11220. https://doi.org/10.1029/2004JA010618

Fairfield, D. H. (1969). Bow shock associated waves observed in the far upstream interplanetary medium. J. Geophys. Res., 74(14), 3541-3553.

https://doi.org/10.1029/JA074i014p03541

Fairfield, D. H. (1974). Whistler waves observed upstream from collisionless shocks. J. Geophys. Res., 79(10), 1368-1378.

https://doi.org/10.1029/JA079i010p01368

Fuselier, S. A., Thomsen, M. F., Gosling, J. T., Bame, S. J., and Russell, C. T. (1986). Gyrating and intermediate ion distributions upstream from the Earth's bow shock. J. Geophys. Res. Space Phys., 91(A1), 91-99.

https://doi.org/10.1029/JA091iA01 p00091

Fuselier, S. A. (1995). Ion distributions in the Earth's foreshock upstream from the bow shock. Adv. Space Res., 15(8-9), 43-52. https://doi.org/10.1016/0273-1177(94)00083-D

Gary, S. P., Gosling, J. T., and Forslund, D. W. (1981). The electromagnetic ion beam instability upstream of the Earth's bow shock. J. Geophys. Res. Space Phys., 86(A8), 6691-6696. https://doi.org/10.1029/JA086iA08p06691

Gary, S. P. (1991). Electromagnetic ion/ion instabilities and their consequences in space plasmas: A review. Space Sci. Res., 56(3-4), 373-415. https://doi.org/10.1007/BF00196632

Gosling, J. T., Asbridge, J. R., Bame, S. J., Paschmann, G., and Sckopke, N. (1978). Observations of two distinct populations of bow shock ions in the upstream solar wind. Geophys. Res. Lett., 5(11), 957-960. https://doi.org/10.1029/GL005i011p00957

Greenstadt, E. W., Le, G., and Strangeway, R. J. (1995). ULF waves in the foreshock. Adv. Space Res., 15(8-9), 71-84. https://doi.org/10.1016/02731177(94)00087-H

Halekas, J. S., Taylor, E. R., Dalton, G., Johnson, G., Curtis, D. W., McFadden, J. P., Mitchell, D. L., Lin, R. P., and Jakosky, B. M. (2015). The solar wind ion analyzer for MAVEN. Space Sci. Rev., 195(1-4), 125-151. https://doi.org/10.1007/s11214-013-0029-z

Hoppe, M. M., Russell, C. T., Frank, L. A., Eastman, T. E., and Greenstadt, E. W. (1981). Upstream hydromagnetic waves and their association with backstreaming ion populations: ISEE-1 and -2 observations. J. Geophys. Res. Space Phys., 86(A6), 4471-4492. https://doi.org/10.1029/JA086iA06p04471

Hoppe, M. M., and Russell, C. T. (1983). Plasma rest frame frequencies and polarizations of the low-frequency upstream waves: ISEE 1 and 2 observations. J. Geophys. Res. Space Phys., 88(A3), 2021-2027. https://doi.org/10.1029/JA088iA03p02021

Hoshino, M., and Terasawa, T. (1985). Numerical study of the upstream wave excitation mechanism: 1 . Nonlinear phase bunching of beam ions. J. Geophys. Res. Space Phys., 90(A1), 57-64. https://doi.org/10.1029/JA090iA01 p00057

Jakosky, B. M., Grebowsky, J. M., Luhmann, J. G., and Brain, D. A. (2015). Initial results from the MAVEN mission to Mars. Geophys. Res. Lett., 42(21), 8791-8802. https://doi.org/10.1002/2015GL065271

Le, G., and Russell, C. T. (1992). A study of ULF wave foreshock morphology - I: ULF foreshock boundary. Planet. Space. Sci., 40(9), 1203-1213. https://doi.org/10.1016/0032-0633(92)90077-2

Le, G., and Russell, C. T. (1994). The morphology of ULF waves in the Earth's foreshock. In M. J. Engebretson, et al. (Eds.), Solar Wind Sources of Magnetospheric Ultra-Low-Frequency Waves (pp. 87-98). Washington: AGU. https://doi.org/10.1029/GM081p0087

Le, G., Chi, P. J., Blanco-Cano, X., Boardsen, S., Slavin, J. A., Anderson, B. J., and Korth, H. (2013). Upstream ultra-low frequency waves in Mercury's foreshock region: MESSENGER magnetic field observations. J. Geophys. Res. Space Phys., 118(6), 2809-2823. https://doi.org/10.1002/jgra.50342

Mazelle, C., Le Quéau, D., and Meziane, K. (2000). Nonlinear wave-particle interaction upstream from the Earth's bow shock. Nonlin. Proc. Geophys., 7(3-4), 185-190. https://doi.org/10.5194/npg-7-185-2000

Mazelle, C., Meziane, K., LeQuéau, D., Wilber, M., Eastwood, J. P., Rème, H., Sauvaud, J. A., Bosqued, J. M., Dandouras, I., .. Balogh, A. (2003). Production of gyrating ions from nonlinear wave-particle interaction upstream from the Earth's bow shock: A case study from Cluster-CIS. Planet. Space Sci., 51(12), 785-795. https://doi.org/10.1016/j.pss.2003.05.002

Mazelle, C., Winterhalter, D., Sauer, K., Trotignon, J. G., Acuña, M. H., Baumgärtel, 
K., Bertucci, C., Brain, D. A., Brecht, S. H., ... Slavin, J. (2004). Bow shock and upstream phenomena at Mars. Space Sci. Res., 111(1-2), 115-181. https://doi.org/10.1023/B:SPAC.0000032717.98679.d0

Mazelle, C., Meziane, K., Wilber, M., and Le Quéau, D. (2007). Wave-particle interaction in the terrestrial ion foreshock: new results from Cluster. AIP Conf. Proc., 932(1), 175-180. https://doi.org/10.1063/1.2778961

McFadden, J. P., Kortmann, O., Curtis, D., Dalton, G., Johnson, G., Abiad, R., Sterling, R., Hatch, K., Berg, P., ... Jakosky, B. (2015). MAVEN SupraThermal and thermal ion compostion (STATIC) instrument. Space Sci. Rev., 195(1-4), 199-256. https://doi.org/10.1007/s11214-015-0175-6

Meziane, K., Mazelle, C., Lin, R. P., Le Quéau, D., Larson, D. E., Parks, G. K., and Lepping, R. P. (2001). Three-dimensional observations of gyrating ion distributions far upstream from the Earth's bow shock and their association with low-frequency waves. J. Geophys. Res. Space Phys., 106(A4), 5731-5742. https://doi.org/10.1029/2000JA900079

Meziane, K., Wilber, M., Mazelle, C., LeQuéau, D., Kucharek, H., Lucek, E. A., Rème, H., Hamza, A. M., Sauvaud, J. A., ... Lundin, R. N. (2004). Simultaneous observations of field-aligned beams and gyrating ions in the terrestrial foreshock. J. Geophys. Res. Space Phys., 109(A5), A05107. https://doi.org/10.1029/2003JA010374

Meziane, K., Wilber, M., Mazelle, C., Parks, G. K., and Hamza, A. M. (2005). A review of field-aligned beams observed upstream of the bow shock. AIP Conf. Proc., 781(1), 116-122. https://doi.org/10.1063/1.2032683

Meziane, K., Mazelle, C. X., Romanelli, N., Mitchell, D. L., Espley, J. R., Connerney, J. E. P., Hamza, A. M., Halekas, J., McFadden, J. P., and Jakosky, B. M. (2017). Martian electron foreshock from MAVEN observations. J. Geophys. Res. Space Phys., 122(2), 1531-1541. https://doi.org/10.1002/2016JA023282

Paschmann, G., Sckopke, N., Bame, S. J., Asbridge, J. R., Gosling, J. T., Russell, C. T., and Greenstadt, E. W. (1979). Association of low-frequency waves with suprathermal ions in the upstream solar wind. Geophys. Res. Lett., 6(3), 209-212. https://doi.org/10.1029/GL006i003p00209

Romanelli, N., Mazelle, C., and Meziane, K. (2018). Nonlinear wave-particle interaction: Implications for newborn planetary and backstreaming proton velocity distribution functions. J. Geophys. Res.: Space Phys., 123(2), 1100-1117. https://doi.org/10.1002/2017JA024691

Russell, C. T., Childers, D. D., and Coleman, Jr. P. J. (1971). Ogo 5 observations of upstream waves in the interplanetary medium: Discrete wave packets. J. Geophys. Res., 76(4), 845-861. https://doi.org/10.1029/JA076i004p00845

Schwartz, S. J., Paschmann, G., Sckopke, N., Bauer, T. M., Dunlop, M., Fazakerley, A. N., and Thomsen, M. F. (2000). Conditions for the formation of hot flow anomalies at Earth's bow shock. J. Geophys. Res. Space Phys., 105(A6), 12639-12650. https://doi.org/10.1029/1999JA000320

Shan, L. C., Lu, Q. M., Wu, M. Y., Gao, X. L., Huang, C., Zhang, T., and Wang, S. (2014). Transmission of large-amplitude ULF waves through a quasi-parallel shock at Venus. J. Geophys. Res. Space Phys., 119(1), 237-245. https://doi.org/10.1002/2013JA019396

Shan, L. C., Mazelle, C., Meziane, K., Delva, M., Lu, Q. M., Ge, Y. S., Du, A. M., and Zhang, T. L. (2016). Characteristics of quasi-monochromatic ULF waves in the Venusian foreshock. J. Geophys. Res. Space Phys., 121(8), 7385-7397. https://doi.org/10.1002/2016JA022876

Sonnerup, B. U. Ö., and Scherble, M. (1998). Minimum and Maximum Variance Analysis. In G. Paschmann, (Ed.), Analysis Methods for Multi-Spacecraft Data (pp. 185-220). Noordwijk, The Netherlands: ESA Publications Division.

Thomsen, M. F., Gosling, J. T., Bame, S. J., and Russell, C. T. (1985). Gyrating ions and large-amplitude monochromatic MHD waves upstream of the Earth's bow shock. J. Geophys. Res. Space Phys., 90(A1), 267-273. https://doi.org/10.1029/JA090iA01p00267

Tsurutani, B. T., Smith, E. J., Burton, M. E., Arballo, J. K., Galvan, C., Zhou, X. Y., Southwood, D. J., Dougherty, M. K., Glassmeier, K. H., ... Chao, J. K. (2001). Oblique '1- $\mathrm{Hz}$ ' whistler mode waves in an electron foreshock: The Cassini near-Earth encounter. J. Geophys. Res. Space Phys., 106(A12), 30223-30238. https://doi.org/10.1029/2001JA900108

Vignes, D., Mazelle, C., Rème, H., Acuña, M. H., Connerney, J. E. P., Lin, R. P., Mitchell, D. L., Cloutier, P., Crider, D. H., and Ness, N. F. (2000). The solar wind interaction with mars: locations and shapes of the bow shock and the magnetic pile-up boundary from the observations of the MAG/ER experiment onboard mars global surveyor'. Geophys. Res. Lett., 27(1), 49-52. https://doi.org/10.1029/1999GL010703

Wilson III, L. B. (2016). Low frequency waves at and upstream of collisionless shocks. In A. Keiling, (Eds.), Low-frequency Waves in Space Plasmas (pp. 269291). Washington: American Geophysical Union. https://doi.org/10.1002/9781119055006.ch16

Winske, D., and Quest, K. B. (1986). Electromagnetic ion beam instabilities: Comparison of one- and two-dimensional simulations. J. Geophys. Res. Space Phys., 91(A8), 8789-8797. https://doi.org/10.1029/JA091iA08p08789

Yamauchi, M., Futaana, Y., Fedorov, A., Frahm, R. A., Winningham, J. D., Dubinin, E., Lundin, R., Barabash, S., Holmström, M., ... Fraenz, M. (2011). Comparison of accelerated ion populations observed upstream of the bow shocks at Venus and Mars. Ann. Geophys., 29(3), 511-528.

https://doi.org/10.5194/angeo-29-511-2011 